\title{
Cavitation Treatment of High-Viscosity Marine Fuels for Medium-Speed Diesel Engines
}

\author{
Sergii Victorovich Sagin ${ }^{1} \&$ Valerii Grigorovich Solodovnikov ${ }^{2}$ \\ ${ }^{1}$ Marine power plants department of Odessa National Maritime Academy, Odessa, Ukraine \\ ${ }^{2}$ Technical operation of the fleet department of Odessa National Maritime Academy, Odessa, Ukraine \\ Correspondence: Sagin Sergii Victorovich, Didrihsona str., 8, Odessa, 65029, Ukraine. Tel: 380-674-821-893. \\ E-mail: saginsergey@mail.ru
}

Received: February 4, 2015

Accepted: February 13, 2015

Online Published: April 7, 2015

doi:10.5539/mas.v9n5p269

URL: http://dx.doi.org/10.5539/mas.v9n5p269

\begin{abstract}
The paper considers processes of treatment for fuels with high sulphur content, when applied in marine medium-speed diesel engines. The paper describes features of operating marine medium-speed diesel engine fuel systems with high-viscosity fuels. The paper offers an option for the cavitation fuel treatment to disrupt the sulphur-carbon bonds in the marine fuels. The authors developed a scheme of the experimental installation, which allows performing cavitation fuel treatment. The results of the analysis are provided in the paper, as well as the fuel cavitation treatment effect on sulphur wear of diesel engine cylinder and piston assembly parts (bushing and top piston ring) is analysed, as well as its operational parameters (maximum cylinder pressure, gases temperature in the exhaust manifold).
\end{abstract}

Keywords: marine medium-speed diesel engine, fuel for marine diesel engines, fuel treatment, sulphur content in the fuel, fuel cavitation treatment, corrosive wear of the cylinder and piston assembly

\section{Introduction}

\subsection{Introduce the Problem}

Marine internal combustion engines are the most common type of heat engines used in marine and river vessels. (Pakhomov Yu. A., 2007). Compared with other types of power plants (gas turbine and steam turbine) used to supply energy to the marine propulsion system, marine diesel engines are characterized by minimum fuel consumption per power unit $(\mathrm{kg} / \mathrm{kW}$.hour) and per path unit traversed by the ship ( $\mathrm{kg} / \mathrm{mile})$. The most widely used are medium-speed diesel engines, used to transmit power over either screw or electric generator (Gershanik C., 2008). In the first case, they serve as the main engines, in the second as the subsidiaries. It shall be noted that the main marine medium-speed diesel engines are used on ships with average displacement, having restrictions on the engine and boiler room size; while they are used as subsidiary (as a part to a ship's power plant) on all ships without exception.

\subsection{Explore Importance of the Problem}

Development trends of marine energy specifically is characterized by the use of high-viscosity fuels with a lower monetary value. The use of such fuels significantly reduces the acquisition cost, which share in total operating costs constitutes up to $80 \%$. Viscosity of such fuels is within $380 \mathrm{sSt}$, and the cost is $450 \ldots 600 \mathrm{USD} /$ tonne depending on the bunkering location. The use of high-viscosity fuels in the marine energy, which began with boiler installations and spread over the crosshead diesel engines model, now is increasingly applied for medium-speed diesel engines. Moreover, current models of these diesel engines and their fuel system are designed specifically for the use with fuels with viscosity up to $380 \mathrm{sSt}$ and sulphur impurities content of up to $3 \%$. At the same time, sea and river fleet operate a sufficient number of earlier versions of diesel engines designed to run exclusively on low-viscosity fuel with sulphur content up to $0.05 \%$. Conversion of these engines to run on high-viscosity sulphur fuels is reduced to the conversion of their fuel equipment. This results in better atomization and further fuel combustion, but does not prevent the phenomenon of sulphurous corrosion of the cylinder and piston assembly. The problems of fuel desulfurization are basically solved at the step of oil refining

(Israelson, G. J., 2004; Dicks, A. L., 1996; Anisimov, A. V.; Tarakanov, A. V., 2008), thus the ships are supplied with fuel, change in the structural composition of which is impossible in the marine environment (Nadkarni, K., 
2000; Wan et al., 2007; Mei et al., 2003).

\subsection{Describe Relevant Scholarship}

The solution to the issue of reducing sulphur engine wear is now fully implemented for low speed diesel engines with separate cylinder lubrication system. At that, the thing remaining is to find an optimal ratio between the sulphur content in the fuel additive and the alkali content in the oil. For example, fuels with a sulphur content of up to $2 \%$ are shall use cylinder oil having a common alkaline index of $40 \ldots 50$ TBN (where TBN - Total Basic Number - the number of alkali milligrams per oil gram); for high-viscosity fuels containing up to $3 \%$ sulphur, TBN value shall be $70 \ldots 80$, and in some cases up to 100 .

Marine medium-speed diesel engines have the general circulation lubrication system, and the use of oils with high alkali content is impractical in this case, both for economic reasons (due to their high cost) and because of their worst operating parameters (in particular less ability to resist normal loads). Therefore, medium-speed diesel engines have the only way to deal with the consequences of using sulphur fuel, which is additional treatment before it enters the cylinder, subsequently contributing to reduced sulphur wear. At that, it is necessary to consider that the solution to remove sulphur impurities from the fuel shall be complex, because any physical effects on the fuel leads to a change in nearly all its components.

Assuming that the weight of dry-and-ash-free fuel contains combustible various sulphur compounds (elemental sulphur, hydrogen sulphide, mercaptans, sulphides, disulphides, thiophenes, theophenes, sulphonic acids, etc.), interacting with the cylinder and piston assemblies, the cylinder head, exhaust and purge valves at different physical and chemical state of the fuel, the following main ways to protect these nodes of diesel engines can be designated:

- manufacture of diesel engine parts of materials resistant to sulphur corrosion;

- changes in the design of diesel engines designed to run on sulphur fuels;

- $\quad$ application of neutralizing anti-sulphur fuel additives;

- exclusion diesel engines operating modes, causing the most intense electrochemical corrosion and wear (i.e. modes with low water temperature, cooling the cylinder walls and the cylinder cover);

- creating fuel treatment systems reducing the number of sulphur compounds entering the diesel engine cylinder;

- operating mode selection for the fuel treatment system providing the best quality of fuel treatment.

The rational use of lubrication and cooling systems also contributes to sulphur wear reduction for the cylinder assembly and the exhaust tract of a diesel engine. Thus, during operation of marine diesel engines, it is necessary to maintain the temperature of the cooling water and oil in the upper range, recommended by the instructions (to avoid condensation of the sulphur combustion products and the formation of sulphur-containing strong mineral acids, primarily sulphurous $\mathrm{H}_{2} \mathrm{SO}_{3}$ and sulphuric $\mathrm{H}_{2} \mathrm{SO}_{4}$ ). When operating the diesel engine at partial load, it is recommended to raise the temperature of the air entering the cylinder by turning off the aftercooler.

\subsection{State Hypotheses and Their Correspondence to Research Design}

One method of fuel treatment, facilitating reduction of the harmful effects of sulphur in the diesel engine cylinder assembly parts, is fuel cavitation treatment with ultrasound. These issues were considered and studied for a relatively low power engines used in road and rail transport (Vasilevskiy, A. V., 2007), in addition, similar studies were carried out for fuels with sulphur content up to $1 \%$ (Promtov, M. A., 2008). Similar studies for marine energy, characterized by high aggregate capacity and battery life, are practically absent. In addition, when determining the relevance of such developments for fuel treatment systems of internal combustion marine engines, one should take into account the risk level of possible failures and complexity of the engineering works in the event of violating a healthy state of a node or an element of the engine. If the delivery of spare parts and their replacement in case of emergency for stationary engines can be accomplished in a few hours, then for marine engines such measures, taking into account the range and autonomy of the ship navigation, can reach several decades of days.

Thus, the introduction of a positive operating experience of additional fuel cavitation treatment systems can improve the reliability and efficiency of marine diesel engines.

\section{Method}

To determine the ultrasonic cavitation fuel treatment effect on piston assemblies wear, as well as on the operational parameters of diesel engines, the laboratory researches were carried out, as well as researches 
directly in the operating conditions of marine diesel engines.

\subsection{Identify Subsections}

The purpose of the laboratory study was to determine the optimal frequency of the acoustic signal for treatment sulphur marine fuels, providing the greatest dispersion of fuel and contributing to the maximum rupture of sulphur-carbon bonds in the fuel.

Marine diesel engines fuel in terms of dispersion system has a viscosity not obeying the laws of Newton, Poiseuille, Stokes, because its long randomly positioned paraffin and resin molecules form a flexible grid. Therefore, the system has a significant resistance to shear forces (Safieva, R. Z., 2004). Cavitation destroys a continuous chain, disrupting bonds between the different parts of the molecules. Thus, cavitation affects the structural change in viscosity, i.e. disrupting the Van der Waals bonds in the fuel. Additional ultrasound fuel treatment primarily improves dispersed qualities of fuel and the cavitation phenomenon accompanying this process leads to further activation of its hydrocarbon constituents and the splitting of C-C and C-S bonds. During the cavitation treatment of hydrocarbon liquids, destruction of molecules occurs, which is caused by micro cracking of molecules and ionization processes. These processes result in system accumulating of "activated" particles: radicals, ions, ion-radical formations (Nesterenko, A. I., \& Berliozov, Yu. S., 2007).

For cavitation effects on the fluid, hydrodynamic, electromagnetic, piezoelectric, magnetostrictive and mechanical cavitation generators are used. The ultrasonic range widely uses piezoelectric and

magnetostrictive cavitation generators. These electroacoustic transducers use direct magnetostrictive and piezoelectric effect in alternating magnetic and electric fields. Transducer excitation frequency range is very wide (from 8 to $44 \mathrm{kHz}$ and above). Ultrasonic vibrations from the transducer are transmitted to the treated substances through special transforming and matching devices (hubs, plates, etc..), ending with the radiating surface (Suslick, K. S., 1989; Khmelev et. al., 2007).

Ultrasonic fuel treatment was performed using UZDN-1 generator with a magnetostrictive transducer.

Dispersity of base and sonicated fuel was evaluated by the nature of its absorption spectrum, which base is the Specord M-40 spectrophotometer (Butenko et al.).

Change in the structural composition of the fuel after ultrasonic cavitation fuel treatment was assessed by analysing its burning spectrum. The essence of this method consists in that line absorption spectra of $\mathrm{H}_{2} \mathrm{~S}$, as one of the components formed upon rupture of C-S bonds of the fuel, were determined by means of flame spectrophotometer. According to the intensity of these spectra, one can judge on the number ${ }^{2} \mathrm{H}_{2} \mathrm{~S}$ bonds formed because of the cavitation process, as a proxy for the fuel desulfurization.

\subsection{Subject Characteristics}

The studies of the cavitation fuel treatment effect on the performance of the marine engines were carried out for a medium-speed diesel engine S6A2 by "Mitsubishi". The main characteristics of diesel engines are as follows: rated power - $420 \mathrm{~kW}$, crankshaft rpm - $750 \mathrm{rpm}$. These diesel engines in the amount of three pieces were part of the marine auxiliary power plant. Each diesel engine had its autonomous fuel system, which made it possible to conduct research for a particular diesel engine with fuel, which passed the different stages of treatment. The fuel system of one of the engines was not retrofitted and was operated in the "normal" mode. At that, the diesel engine was taken as a "reference" one. Fuel to the other two diesel engines was supplied after additional cavitation treatment, for which the fuel system of these diesel engines was retrofitted with ultrasonic cavitation treatment module. Fragment area for retrofitting diesel engines fuel system is shown in Figure 1. 


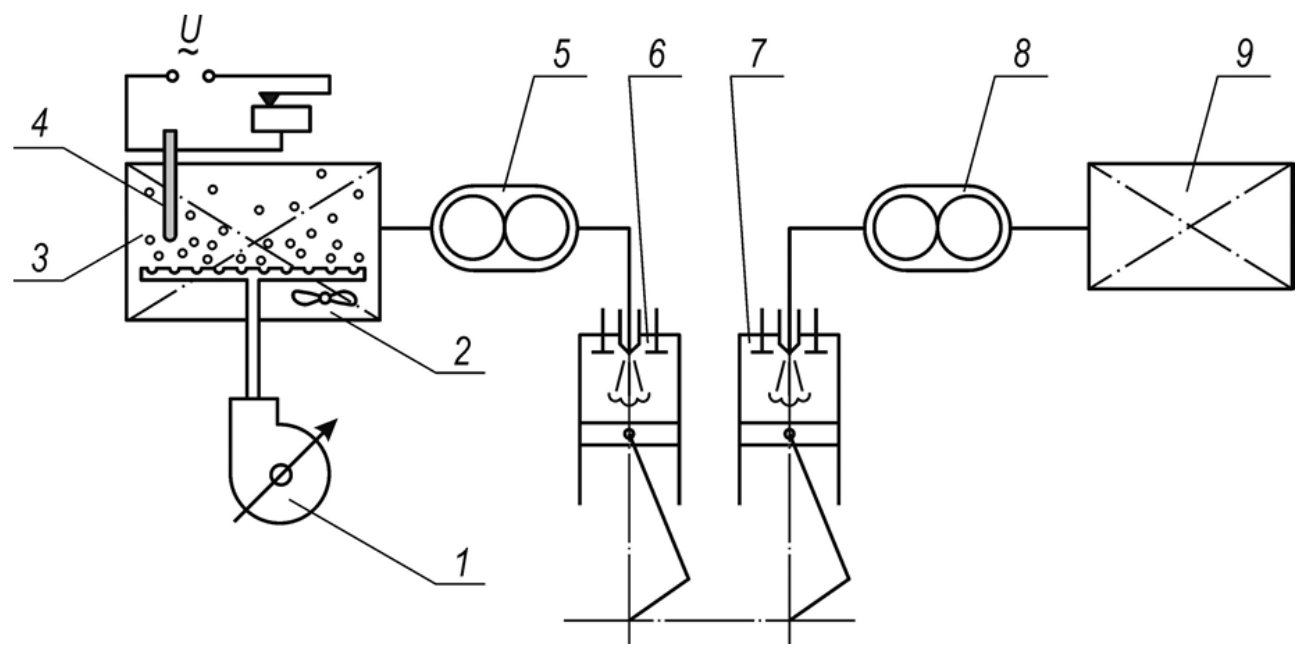

Figure 1. Retrofitting diagram of supplying fuel to the engine (detail)

1 - air compressor; 2, 9 - fuel tanks; 3 - air line; 4 - ultrasonic installation; 5, 8 - fuel pumps; 6, 7 - engines.

Engine 7 was the reference sample and was operated in "normal" mode, at that, fuel passing the standard preparation, was supplied by pump 8 from the tank 9 . Engines 6 were "experimental samples". Fuel was pumped 5 from the tank 2 in which the fuel was exposed to ultrasonic waves generated by the UZDN-1 generator with magnetostrictive radiator. At the same time, to enhance the cavitation, additional airline 3 was constructed at the bottom of one of the tanks. The air compressor 1 provided air injection in the line. At that, the air bubbles entering the amount of fuel were an additional source of cavitation zones.

Ultrasonic installation 4 was operated in the mode $I=0.4$ A using magnetostrictive transducer $22 \mathrm{kHz}$. These values were identified in previous laboratory studies and provided the best dispersed performance of the fuel (Sagin, S. V., Solodovnikov, V. G., 2014). Paddle mixer providing a uniformity of fuel throughout the volume, was installed in the fuel tank 2, in which the fuel passed the ultrasonic treatment. Fuel NFO380 was used for the experiments of diesel engines.

\subsection{Sampling Procedures}

In order to ensure the equivalence of the experiment, their preliminary preparation for tests was carried out for all diesel engines. Operating conditions allowed 40 sequential hours of performing a full engine purge these diesel engines. At the same time, all diesel engines had piston assembly (pistons and piston rings) and the basic elements of the high-pressure fuel system (precision pairs of high-pressure fuel pump - plunger-bushing and injector needle-injector nozzle) replaced. In addition, before the start of the experiments, both engines were subject to control and regulation of the fuel equipment. At that, high-pressure fuel pumps were regulated by the same angle for advancing fuel supply and engines' injectors were tuned to the same pressure for the needle lift.

During the whole period of the experiment, operating time and operational stress on diesel engines were monitored. To achieve a uniform distribution of operation time, diesel engines were consistently put on stand-by mode. According to the technology of the experiment, the time difference for diesel engines operation, as well as the operational loads on the engines did not exceed $4 \%$, which, given the energy-intensive facilities, allowed to consider their working conditions as identical. The engines were operated at the same fuel type. At that, the means of automatic control supported fuel viscosity constant throughout the experiment. The grade of circulating oil providing lubrication modes and its performance were identical throughout the experiment. These measures allowed supposing that the execution of the experiment took place in similar conditions.

The task of the study was to determine the wear of cylinder bushings and piston top ring for a "standard" fuel, and fuel subjected to the additional cavitation treatment.

Determination of cylinder bushing wear was made near of the top piston ring, because it is an area most exposed to sulphur wear and sulphur corrosion. To improve the accuracy and reliability of measurements, the determination of cylinder bushings wear was made by artificial databases and indicator measurement methods (with an accuracy to $0.01 \mathrm{~mm} / \mathrm{m}$ ). Deviation in determining wear by these methods did not exceed $7 \%$, which confirms the correctness of measurements (Solodovnikov, V. G., 2014). Piston rings wear was performed by weighing on electronic scales. Prior to the measurement of cylinder bushings and piston rings weighing, the 
samples were subject to preliminary cleaning from soot particles formed on their surfaces. For this purpose, their surface was washed with MDO diesel fuel.

Wear measurements were performed for the two outer cylinders known to be subject to this phenomenon more than the other (central cylinders). At that, the resulting values were averaged. In addition, the study of only two cylinders significantly reduced the installation work time restricted by the terms of use. For the same reasons, the studies were carried out after 180, 390, 590, 810, 1030 hours of engines operation.

As the performance of diesel engines operation, we determined the temperature of gases before gas turbo blower and the maximum combustion pressure, which were measured using the marine diagnostics and control means. These parameters are related to the primary parameters, used for regulating diesel engine workflow and definition of the load mismatch for cylinders. Moreover, the data of the value determine thermal and mechanical stresses of diesel engines.

\section{Results}

\subsection{Determination of Sulphur Wear for Cylinder Assembly Elements}

During the combustion of fuel in the diesel engine cylinder, all sulphur compounds contained in it form sulphur dioxide SO2 and trioxide SO3 (Assad, M. S., \& Penyazkov, O. G., 2010). Simultaneously, a large amount of water vapours forms in the cylinder and thus, a system of two condensable components $\mathrm{H} 2 \mathrm{O}$ - $\mathrm{H} 2 \mathrm{SO} 4$ occurs in the combustion products, providing the possibility of intense sulphur corrosion, which may be gaseous or liquid. Sensitivity of diesel engines to sulphur corrosion depends on the intensity of the heat stress at the sleeve assembly and specific performance. If there is oxygen excess and temperature is high, thermal stress and the gas corrosion intensity increase. This leads to gas corrosion of the vent valve plates, upper cylinder bushing, the first piston ring and the blowoff path. When reducing the temperature in the diesel engine cylinder, the possibility of this type of corrosion and associated wear is reduced. At a sharp decrease in the thermal regime, liquid corrosion prevails, increased intensity of which occurs during engine start-up. Both gas and liquid sulphur corrosion is the cause of sulphur presence in fuel composition.

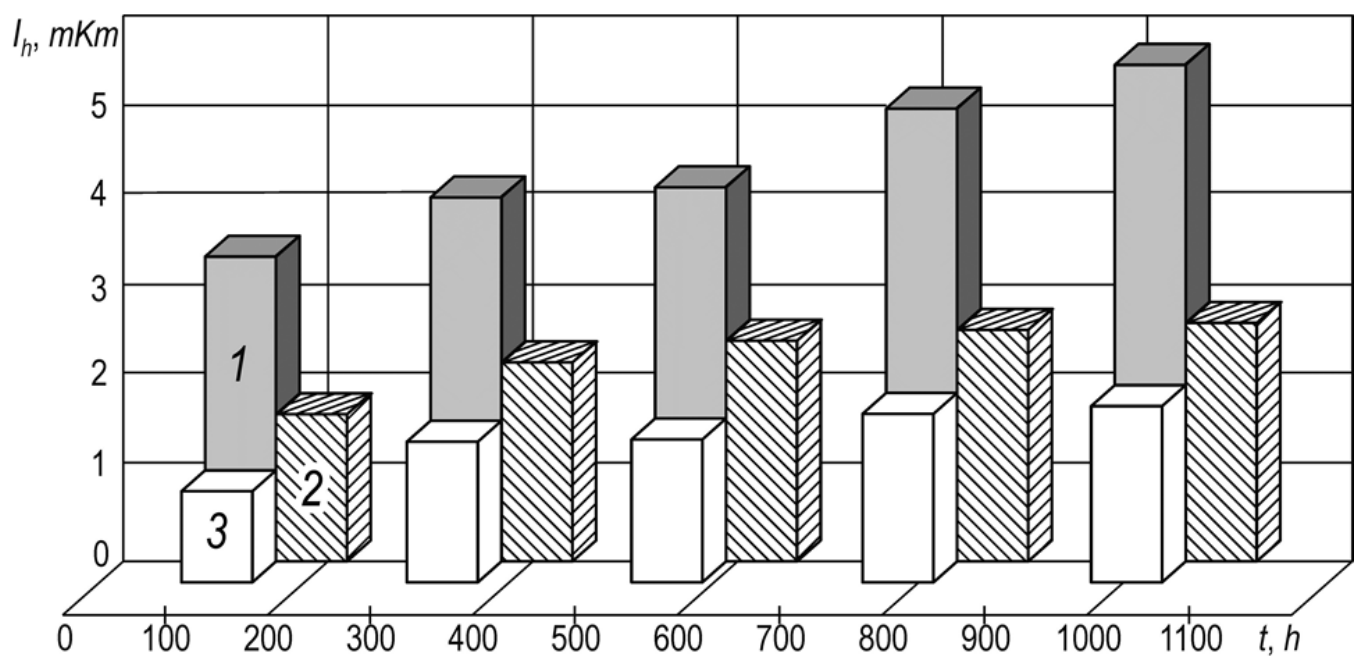

Figure 2. Corrosion wear of cylinder bushings of the S6A2 marine medium-speed diesel engine by "Mitsubishi" under different experimental conditions:

1 - fuel without additional treatment (at operation of diesel fuel treatment system in "normal" mode);

2- fuel elapsed additional cavitation treatment (with the additional use of ultrasonic cavitator only in the fuel treatment);

3 - fuel elapsed additional cavitation treatment (using an ultrasonic cavitator and additional air supply to the cavitation zone)

At cavitation fuel treatment, it is ruptured under tensile stress, the epicenter of which is the so-called "cavitation cavity". At the moment of rupture of the cavitation cavity, gas pressure and temperature may locally reach 
significant values (as calculated up to $100 \mathrm{MPa}$ and up to $10000 \mathrm{~K}$, respectively). After the rupture of the cavity, the spherical shock wave, rapidly attenuating in space, distributes in the surrounding fluid. When generating impulse tensile stresses in the fluid, cavitation nuclei present therein (stable steam and gas bubbles of small size) begin to rise, forming a cavitation cluster, the shape and the initial size range of cavitation nuclei, the applied stress and the nature of the boundary conditions determines dimensions of which. Fluid vapours, dissolved gases and substances with a high vapour pressure can penetrate into a cavitation cavity, while ions or molecules of non-volatile solutes cannot (Knapp et al., 1974, Rozhdestvenskiy, V. V., 1977). The energy, released during the cavity collapse, is sufficient for excitation, ionization and dissociation of sulphur, which subsequently leads to rupture of C-S bonds.

Effect of cavitation fuel treatment on sulphurous corrosion of cylinder assembly can be analysed to determine the wear of cylinder bushing and the top piston ring. The results of measuring the wear of cylinder bushings and piston rings are shown in Figure 2 and 3.

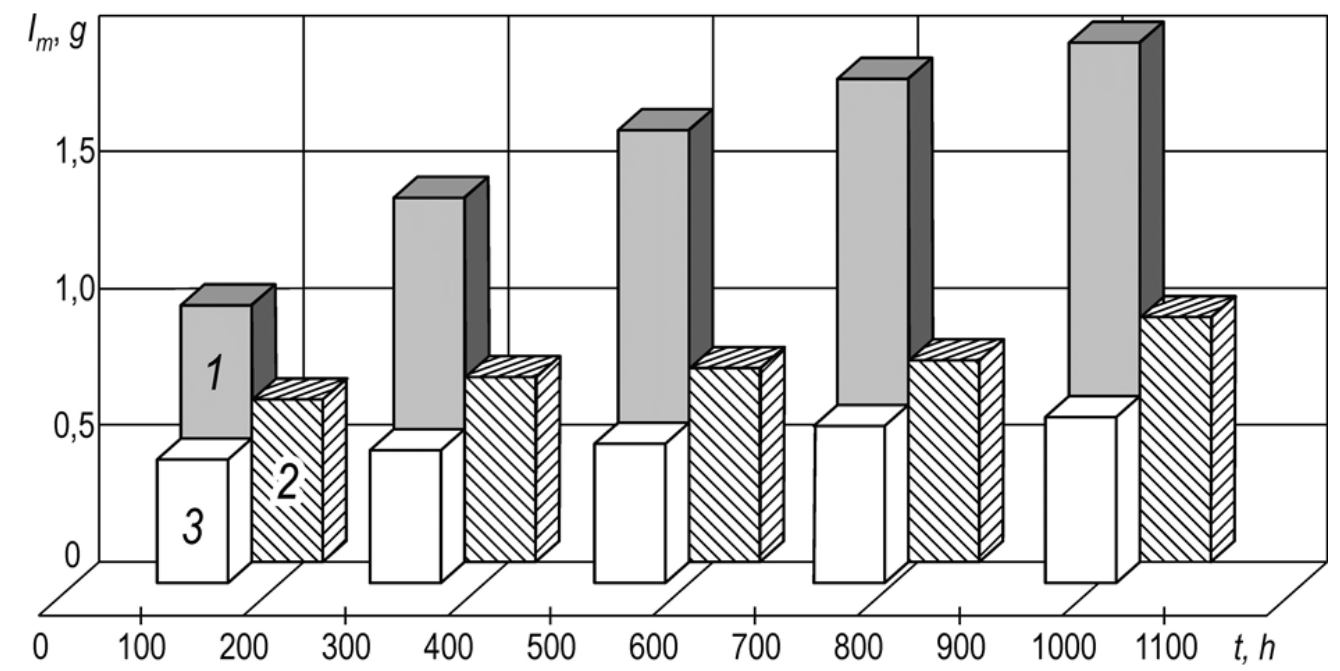

Figure 3. Corrosion wear of piston rings of the S6A2 marine medium-speed diesel engine by "Mitsubishi" under different experimental conditions

1 - fuel without additional treatment (at operation of diesel fuel treatment system in "normal" mode);

2- fuel elapsed additional cavitation treatment (with the additional use of ultrasonic cavitator only in the fuel treatment);

3 - fuel elapsed additional cavitation treatment (using an ultrasonic cavitator and additional air supply to the cavitation zone).

\subsection{Determination of Operating Parameters for the Diesel Engine}

The results of thermal studies, which determined the maximum cycle pressure and temperature of the gases in the exhaust manifold, are shown in Figure 4 and 5. 


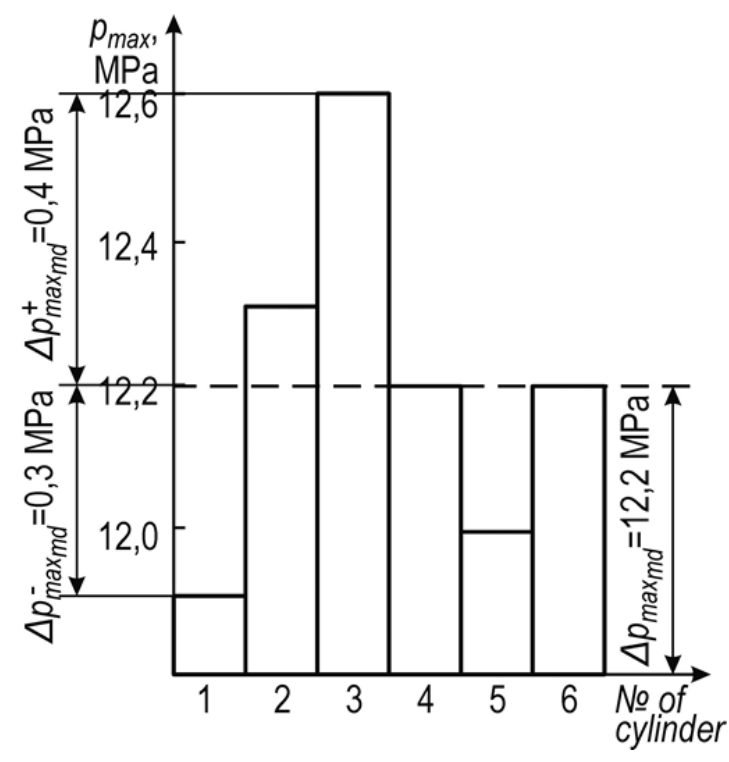

a)

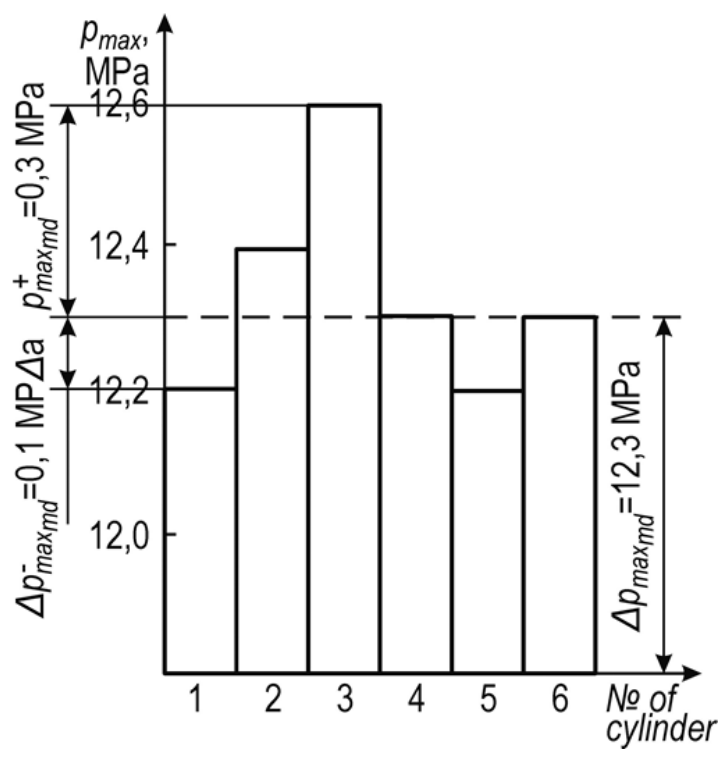

b)

Figure 4. Mismatch of maximum cycle pressure over the cylinders of the S6A2 marine medium-speed diesel engine by "Mitsubishi" under different experimental conditions

a) fuel without additional treatment (at operation of diesel fuel treatment system in "normal" mode);

b) Fuel elapsed additional cavitation treatment (using an ultrasonic cavitator and additional air supply to the cavitation zone).

High-energy intensity and saturation of the fuel system of marine diesel engines are characterized by the most responsible in terms of reliability, tribounit - the plunger-bushing friction pair of high-pressure fuel pump and the friction pair injector needle - injector body. Therefore, during the experiments, we evaluated the technical condition of fuel equipment of a diesel engine, and, in particular, the surface condition of the fuel pump plunger. The main type of defects in this unit is its wear. However, given the degree of fuel filtration, one can state that parts of high-pressure fuel equipment are more prone to corrosion wear than to mechanical or abrasion wear. Thus, the presence of water in the fuel corrode surfaces of precision pairs elements, while significantly enhancing with increasing amounts of sulphur compounds in the fuel.

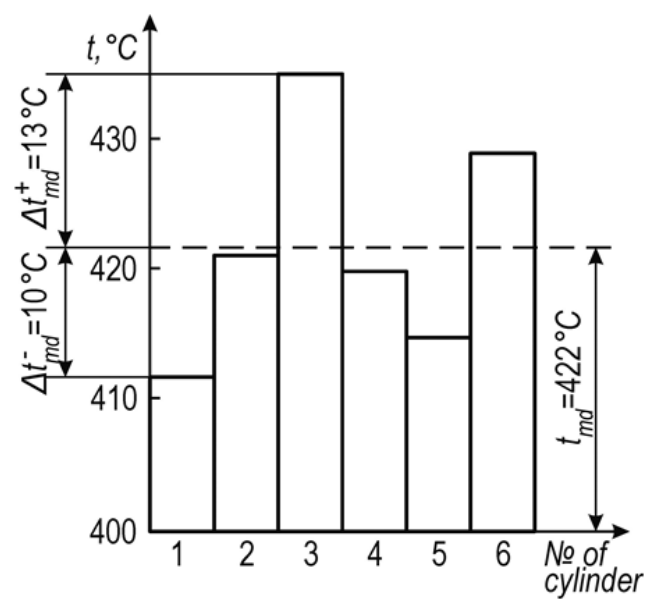

a)

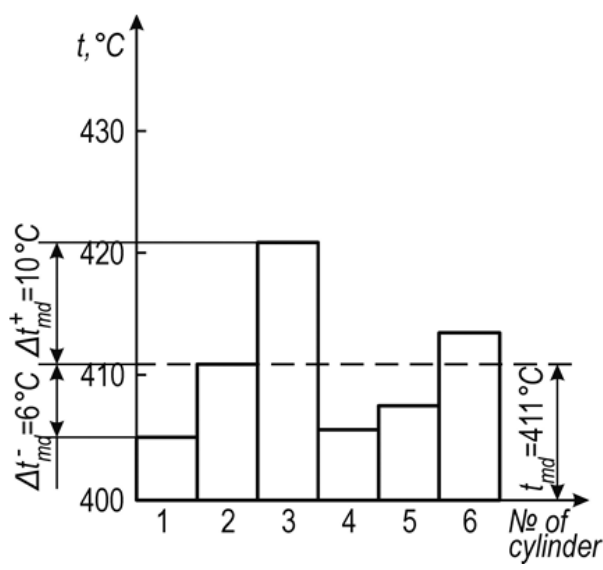

b)

Figure 5. Mismatch of gas temperature in the exhaust manifold over the cylinders of the S6A2 marine medium-speed diesel engine by "Mitsubishi" under different experimental conditions:

a) fuel without additional treatment (at operation of diesel fuel treatment system in "normal" mode); 
b) Fuel elapsed additional cavitation treatment (using an ultrasonic cavitator and additional air supply to the cavitation zone).

Particular form of corrosive wear is oxidation, a process of formation of oxide films on the surfaces of elements due to the adsorption of oxygen dissolved in the fuel. During friction process, the oxide films are destroyed, creating wear products, consisting of metal oxides. This type of wear is usually observed in the boundary lubrication conditions (typical for fuel injection equipment) when there is a local contact of surfaces, accompanied by plastic deformation and saturation of the metal surface layers with oxygen dissolved in the fuel. With increasing thickness of the oxide films, the latter become brittle and internal stress and also under the influence of pressure shocks the fuel jets, their destruction occurs, and the portions of the underlying metal surface are exposed and the destruction process repeats.

Assessment of the high-pressure fuel pump plunger surface wear is determined by the area of visible wear spots $S_{\text {Ih }}$. The plungers of the two outer fuel pumps (providing the operation of the 1st and 6th cylinder) were dismantled in the range of operation of a diesel engine, allowing short-term decommissioning and equal to 220 , 410, 640, 830 and 1060 hours to perform the above-mentioned operation. After that, in the marine laboratory performed determination of $S_{\mathrm{Ih}}$ using microscopic studies. The technology of measuring the plunger surfaces corresponds to the previously described method on determination of the cylinder bushings wear. At that, in addition to clean the surfaces with ether or refrigerant, which ensured the absence of oxide films at the surface and increased accuracy of the measurement. Wear dynamics for the plunger-bushing precision pair for high pressure fuel pump is shown in Figure 6.

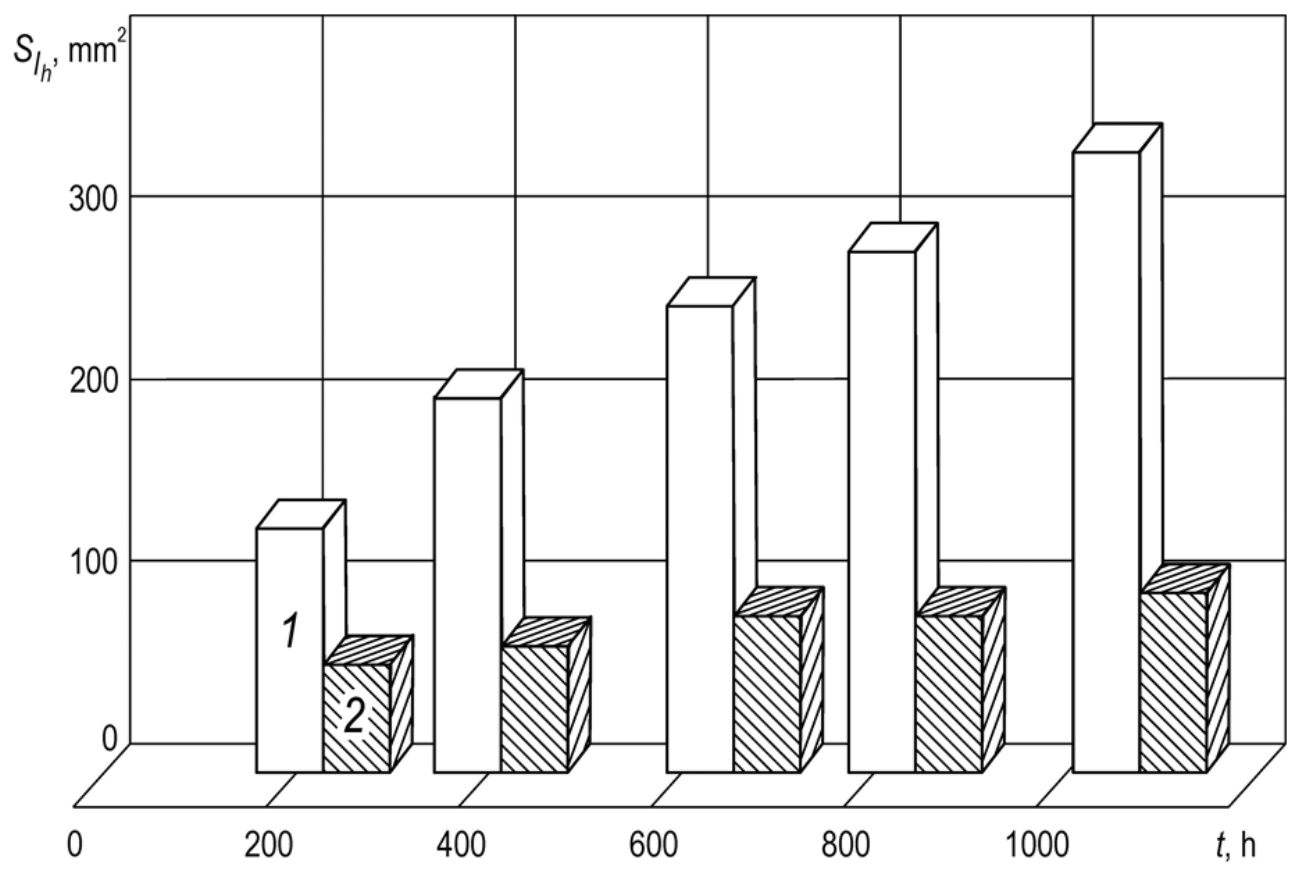

Figure 6. Dynamics of changes in wear of the plunger-bushing precision pair of high pressure fuel pump of the

S6A2 medium-speed marine diesel engine by "Mitsubishi" under different experimental conditions

1 - fuel without additional treatment (at operation of diesel fuel treatment system in "normal" mode);

2 - Fuel elapsed additional cavitation treatment (using an ultrasonic cavitator and additional air supply to the cavitation zone).

\section{Discussion}

The experimental study shows that cavitation fuel treatment leads to a reduction of cylinder and piston assembly sulphur wear. At that, the greatest decrease in this parameter was observed for piston rings, which is especially important, given the importance of the unit in ensuring the quality of such processes as compression, combustion 
and expansion, and the reliability of the piston - cylinder-bushing relation.

Reduced cylinder bushings (by 49.1...65.4\%) and the top piston ring (by 51.5...71.9\%) sulphur wear confirms the appropriateness of cavitation treatment for marine fuel, containing sulphur impurities in its composition.

Additional compressed air supply into the cavitation zone while cavitation treatment promotes intensification of the process, as evidenced by the further deterioration of determining value of sulphur wear; it is consistent with the results of other studies conducted in this area (Nero et al., 2002, Field, K., 2004, Zaporozhets et al., 2004).

The results obtained when determining the operating parameters for the diesel engine operation (maximum cycle pressure and temperature of the gases in the exhaust manifold) indicate a reduction of both mechanical and thermal stresses on diesel engine while using sulphur fuel with preliminary cavitation treatment. According to the requirements of the Operating and Maintenance Rules for Marine Technical Equipment, the mismatch of the value data in the diesel engine cylinders from the mean value shall not exceed $\pm 3.5 \% \Delta p_{\max }$ and $\pm 10^{\circ} \mathrm{C}$. Decreasing the interval in the case of using the cavitation fuel treatment reduces dynamic loads on the diesel engine crank mechanism and diesel engine rotation speed unevenness. Both of these parameters, in turn, reduce the bending and twisting moments on the diesel engine crankshaft. In addition, the increased degree of the maximum cycle pressure deviation in the diesel engine cylinders (which is typical for fuel without further cavitation treatment) witnesses both the increase of the tribological losses in diesel engine fuel equipment elements, and the worsened fuel atomization.

Cavitation fuel treatment (as confirmed experimentally) also contributes to reduced wear of the plunger-bushing precision pair of high-pressure fuel pump. In operating conditions, it contributes to reduction of fuel leakage through this tribocoupling and intensification of the process of injecting fuel into the diesel engine cylinder.

Thus, the obtained results support the use of cavitation treatment of high-viscosity sulphur fuels as an additional method for fuel processing, contributing to both reducing cylinder and piston assemblies sulphur wear and improved operational parameters of the marine medium-speed diesel engines in their operation on high-viscosity fuels with high sulphur content.

\section{Acknowledgments}

The foregoing studies were conducted in accordance with the implementation plan for research works of Odessa National Maritime Academy on the following topic: "Development of systems and methods to improve the technical operation of ship power plants based on modern information technology". The authors express their gratitude to the Vice-Chancellor for Science of Odessa National Maritime Academy, Doctor of Technical Sciences, and Professor Golikov Vladimir Antonovich for assistance in organizing experimental work and recommendations on the design of research results.

\section{References}

Anisimova, A. V. (2008). Oxidative desulfurization of hydrocarbons. Ros. Chem. Journal (Journal Ros. Chem. Community N.A. D. I. Mendeleev), T. LII(4), 32-40.

Assad, M. S. (2010). Products of liquid and gaseous fuels combustions: Formation, calculation, experiment. Minsk: Belarus. Navuka, 305.

Dicks, A. L. (1996). Hydrogen generation from natural gas for the fuel cell systems of tomorrow. Journal of Power Sources, 61, 113.

Field, K. (2004). Low Sulfur Fuel Analysis - Chasing the Limits. K. Field. Petro Industry News.

Gershanik, C. (2008). Optimizing main engine running mode to decrease fuel consumption of seagoing vessels. Journal of Marine Engineering \& Technology (JMET), 7(2), 33-42.

Israelson, G. J. (2004). Results of testing various natural gas desulfurization adsorbents. Journal of Materials Engineering and Performance, 13, 282.

Knapp, R., Cavitation, R., Knapp, D. J., \& Hammit, F. (1974). M.: Mir, 668.

Mei, H., Mei, B. W., \& Yen, T. F. (2003). Fuel, 82(4), 405-414.

Nadkarni, K. (2000). Determining Trace Amounts of Sulfur in Petroleum Products. World Refining.

Nero, V. P. (2002). Oxidative process for ultra-low sulphur diesel. Preprints of Papers. American Chemical Society, Division of Petroleum Chemistry.

Nesterenko, A. I. (2007). The possibility of hydrocarbons cracking under cavitation. Quantitative energetic assessment. Berliozov. Chemistry and Technology of Fuels and Oils, 6, 43-44. 
Pakhomov, Y. A. (2007). Ship power plants with internal combustion engines (p528). M.: TransLit.

Promtov, M. A. (2008). Prospects for using cavitation technologies for chemical-engineering processes intensification. TGTU Bulletin, 14(4), 861-869.

Rozhdestvenskiy, V. V. (1977). Cavitation. L.: Shipbuilding, 248.

Safieva, R. Z. (2004). Chemistry of oil and gas. Oil dispersion systems: Composition and properties (part 1). Textbook. M.: RSU of Oil and Gas n.a. I. M. Gubkin.

Sagin, S. V. (2014). Ultrasonic fuel treatment for reducing sulphur engine wear. Alm. of Art. under the Materials of XXXV Intern. Scientific and Practical conf. Engineering sciences - from theory to practice, 6(31), 42-49. Novosibirsk: SibAK.

Solodovnikov, V. G. (2014). Various fractional and structural fuel composition in marine diesel engines. Ship power plants, 33, 52-58. Odessa.

Suslick, K. S. (1989). The chemical effects of ultrasound. Scientific American (pp. 80-86).

Ultrasonic multifunctional and specialized devices for intensification of technological processes in industry, agriculture and household. V.N. Khmelev and others. Biisk: Publ. house of Alt. State technical Univ. -2007. $-400 \mathrm{p}$.

Vasilevskiy, A. V. (2007). The method to improve the starting characteristics of diesel engines at low temperatures by means of ultrasonic cavitation treatment of diesel fuel. Materials from international scientific and engineering conf. AAI International Conference "Automobile and Tractor Industry in Russia: Priorities of Development and Personnel Education" dedicated to the 145th anniversary of MAMI MSU. Section 2 "Piston and turbine engines". P. 60-66.

Wan, M. W., \& Yen, T. F. (2007). Appl. Catal. A: General, 319, 237-245.

Zaporozhets, E. P. et al. (2004). The study of vortex and cavitation flows in hydraulic systems. Theoretical Foundations of Chemical Engineering, 38(3), 243-252.

\section{Copyrights}

Copyright for this article is retained by the author(s), with first publication rights granted to the journal.

This is an open-access article distributed under the terms and conditions of the Creative Commons Attribution license (http://creativecommons.org/licenses/by/3.0/). 